\title{
Spectra of North Pacific Temperature and Salinity Perturbations in the Depth Domain
}

\author{
GuNNAR I. RODEN \\ Dept. of Oceanography, University of Washington, Seattle
}

(Manuscript received 18 March 1970, in revised form 15 April 1970)

\begin{abstract}
In the central North Pacific, temperature and salinity depth profiles show considerable fine structure, the features of which are most pronounced in the thermocline and halocline, as well as near water mass boundaries. By partitioning the observed series into mean and perturbation values, it is possible to obtain some statistical measures for the perturbations. Wavenumber spectra of temperature, salinity, sound velocity and density were computed for the wavenumber range between 0 and 167 cycles per kilometer. The perturbation spectra for the different geographical regions of the transition zone are essentially the same, for a given variable. In all cases the power density of the perturbations decreases with increasing wavenumber. The power law decay coefficient at low wavenumbers is about $-8 / 3$. It decreases gradually with increasing wavenumber. Superimposed upon the smooth decrease in power density are random fluctuations, most of them insignificant at the $95 \%$ confidence level. The perturbations are generally incoherent over distances of the order of $50 \mathrm{~km}$. For a given station, temperature and salinity perturbations are coherent at low wavenumbers, but incoherent at high wavenumbers.
\end{abstract}

\section{Introduction}

In April 1968, the Research Vessel Thomas G. Thompson made a detailed study of the vertical temperature and salinity structure in the central North Pacific. Three meridional sections of closely spaced stations were occupied between latitude $45 \mathrm{~N}$ and the Hawaiian Islands, traversing the transition zone between the subarctic and subtropical water masses (Roden, 1970). The records cover the upper $1500 \mathrm{~m}$ of the ocean and show considerable fine structure. It is of interest to consider what oceanographically useful information can be extracted from the records by modern statistical means.

At any fixed geographical location, temperature and salinity are complicated functions of depth and time involving periodic as well as random components. The periodic components are chiefly astronomical in origin, resulting from the interaction of tidal flow with the geometry of ocean basins. The random components are mostly meteorological in origin and reflect the effect of "weather" upon the temperature and salinity fields.

The temperature and salinity fluctuations in time and with depth are intimately related to non-uniform flow in a stratified medium. For a given flow velocity, the amplitude of the fluctuations will be large where the vertical temperature and salinity gradients are strong, and small where the gradients are weak. Thus, the size of the fluctuations is likely to be more pronounced in the thermocline and halocline and near water mass boundaries than elsewhere. This is fully confirmed by observations, as will be shown later.
Of the periodic components, those studied involve principally temperature fluctuations of tidal frequency (Reid, 1956; Fjeldstad, 1964; Cairns and LaFond, 1966; Lee and Cox, 1966), inertial frequency (Munk and Phillips, 1968; Webster, 1968), and diurnal and annual temperature changes (Koizumi, 1955; LaFond, 1962; Tabata, 1965). Other regular components have been postulated on theoretical grounds (Rattray, 1964; Rattray and Charnell, 1966) and await verification by actual measurement.

The irregular fluctuations are often attributed to turbulence, and attempts have been made to explain the shape of the spectrum of the fluctuations in terms of classical homogeneous turbulence theory. This theory, critically reviewed by Saffman (1968), predicts a $-5 / 3$ power law for the relation between spectral energy and wavenumber. There is some indication that the $-5 / 3$ power law holds for high wavenumbers in the ocean (Grant et al., 1962) and in the atmosphere (MacCready, 1962). At low wavenumbers, on the other hand, the observed energy decrease is much stronger than expected from the $-5 / 3$ power law (Stewart and Grant, 1962). The failure of the classical homogeneous turbulence theory at low wavenumbers may be attributed to stratification effects in a gravitational field (the buoyancy force), which renders turbulence highly anisotropic (Bolgiano, 1962): Lumley (1964) and Phillips (1966) have investigated from a theoretical point of view turbulence in a stratified medium and have predicted $\mathrm{a}-11 / 5$ and $\mathrm{a}-3$ power law for the decrease in spectral energy of velocity with increasing wavenumber. There is little observational evidence to 
prefer one power law over the other; Myrup (1968) found that his observations fell between the theoretically predicted slopes.

The information now available on the vertical and horizontal salinity structure in the ocean is fragmentary, because few observations have been made with continuously recording instruments in the space domain. An analysis of temperature inversions in the upper 300 $m$ of the ocean, based on bathythermographs, showed that the most prominent of these were concentrated at water mass boundaries and had characteristic thicknesses of 20-50 m (Roden, 1963). LaFond (1963), using a temperature structure profiler (a $274 \mathrm{~m}$ chain with sensors placed at approximately $8 \mathrm{~m}$ intervals), was able to establish that the characteristic horizontal extent of temperature inversions was of the order of $1 \mathrm{~km}$ or less off Baja California. Lovett (1968) studied the vertical temperature gradient structure and attributed the latter to shear flow and Kolmogorov turbulence.

\section{Data}

The following analysis is based on data obtained by the Bissett-Berman electronic salinity-temperaturedepth (STD) sensor. The instrument has a temperature accuracy of $0.01 \mathrm{C}$, a salinity accuracy of $0.03 \%$ and a depth accuracy of $0.25 \%$ of the actual value. The STD device was lowered from the surface to $1500 \mathrm{~m}$ at a rate of $90 \mathrm{~m} \mathrm{~min}^{-1}$, yielding measurements every $3 \mathrm{~m}$, on the average. The digitized data were passed through an error-screening routine and through a linear interpolation scheme to yield values at exactly $3 \mathrm{~m}$ intervals.

The equally spaced series were further partitioned into mean series and perturbation series consisting of departures from the mean. Denoting the original series by $s(z)$, the mean series $s^{*}(z)$ was obtained from

$$
s^{*}(z)=\sum_{i=-m / 2}^{m / 2} w(i) s(z+i)
$$

where $2 v(i)$ is a suitable smoothing array satisfying the conditions

$$
w(i)=w(-i), \quad \sum_{i} w(i)=1 .
$$

The choice of $w(i)$ depends upon the problem one wants to investigate. A sufficiently general class of smoothing arrays can be derived from a modified binominal sequence

$$
w(i)=\left[\frac{m !}{(m-i) ! i !}\right]^{p} / \sum_{i}\left[\frac{m !}{(m-i) ! i !}\right]^{p},
$$

where $m$ is even and $p$ is an arbitrary exponent. The parameters $m$ and $p$ control the width and peakedness of the smoothing array. For $p=1$, the set of ordinary binomial weighting coefficients is obtained. The larger $m$, the greater is the degree of smoothing; but too large an $m$ shortens the original series by an unacceptable amount, leading to a loss of relevant information. Thus, a compromise must be reached between the degree of smoothing and the retention of important information. After examining the smoothing characteristics of several filters of type (3) and the observed depth distribution of temperature and salinity, it was found that the minimum value of $m$ to yield a statistically stable mean series was 40 , with $p=0.5$.

The perturbation series was obtained by subtracting the smoothed series from the original series. This method of high-pass filtering has the response

$$
r(k)=1-\sum_{i} w(i) \cos (2 \pi k \Delta l),
$$

where $k$ refers to the wavenumber and $\Delta l$ is the digitizing interval. The response function for the modified binomial filter used in the subsequent analysis $(m=40$, $p=0.5, \Delta l=0.003 \mathrm{~km}$ ) is shown in Fig. 1 .

\section{Nature of perturbations in the depth domain}

Typical perturbation series for temperature, salinity, and several derived quantities are shown in Fig. 2. The outstanding feature is that large perturbations are confined to a limited depth interval, here between about 200 and $600 \mathrm{~m}$. In the uppermost layers of the sea, mixing and diffusion effectively eliminate smallscale structural features in the temperature and salinity field. At great depths, the amplitude of the temperature and salinity perturbations is also small, because of sluggish motion and the smallness of horizontal and vertical gradients. Hence, a region of increased amplitude perturbations is likely to lie between the surface and deep layers of the ocean. In fact, at all stations in the transition zone, the largest perturbations occurred in and slightly below the thermocline and halocline.

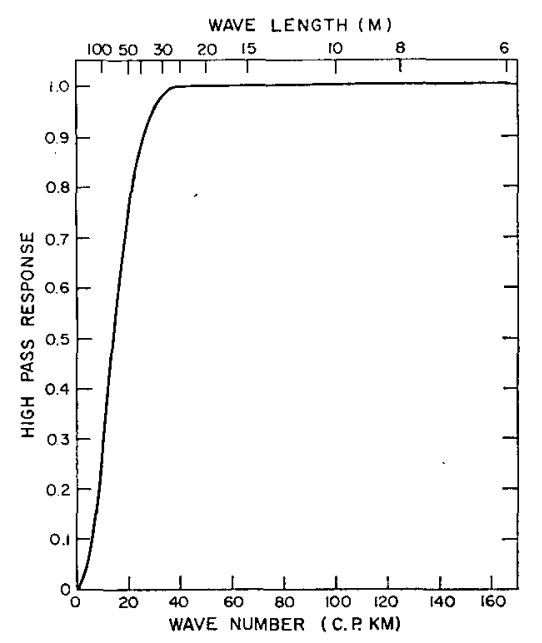

FlG. 1. High-pass response function of binomial filter. 
This seems to be an inherent characteristic of the stratified ocean.

It is of interest to look briefly into the vertical length scales and amplitude values of the perturbations. The mean vertical length scales, as obtained from an analysis of zero-crossing intervals of records such as shown in Fig. 2, are given in Table 1. Temperature perturbations are, on the average, $13-16 \mathrm{~m}$ thick. Sound velocity perturbations have a similar thickness, as would be expected from the close relationship between sound velocity and temperature. Salinity perturbations have a mean thickness of $8-10 \mathrm{~m}$, and $\sigma_{s, t, p}$-density perturbations are, on the average, $8-10 \mathrm{~m}$ thick. There is very little difference between the northern and southern regions of the transition zone.

The amplitudes of the perturbations vary with depth, as has been mentioned above. The largest amplitudes for each variable are listed in Table 2. For temperature, the maximum amplitudes vary typically between 0.1 and $0.2 \mathrm{C}$, with occasional higher values near the southern boundary. For salinity, the maximum amplitudes range from $0.03-0.11 \%$, with the higher values in the southern region of the transition zone. For sound velocity, the largest amplitudes vary from $0.2-1.1 \mathrm{~m}$ $\sec ^{-1}$, with the higher values near the boundaries. The maximum amplitudes of the $\sigma_{s, i, p}$-density range from $0.02-0.07 \mathrm{mg} \mathrm{cm}^{-3}$, and are more pronounced in the south than they are in the north.

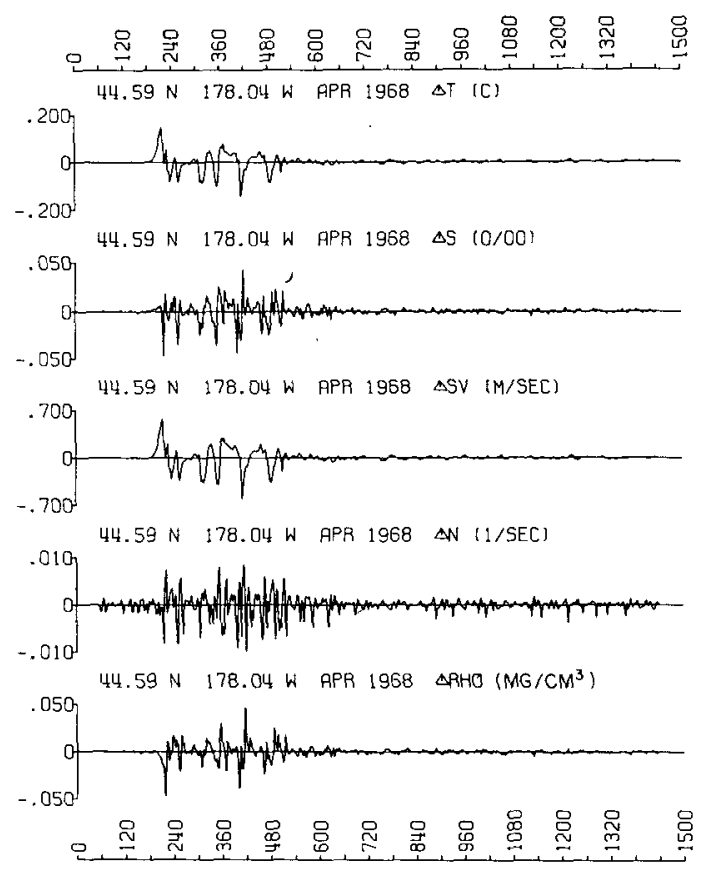

FIG. 2. Perturbations from the mean as a function of depth (m), obtained by binomial filtering: $T$ temperature; $S$ salinity, $S V$ sound velocity, $N$ the Väisälä frequency, and RHO the $\sigma_{s, t, p}$ density.
TABLE 1. Mean vertical length scales of the perturbations $(\mathrm{m})$.

\begin{tabular}{|c|c|c|c|c|}
\hline & Temperature & Salinity & $\begin{array}{c}\text { Sound } \\
\text { velocity }\end{array}$ & $\sigma_{s, t, p}$-density \\
\hline \multicolumn{5}{|c|}{ Northern region } \\
\hline $\begin{array}{l}43 \mathrm{~N}, 178 \mathrm{~W} \\
42 \mathrm{~N}, 178 \mathrm{~W} \\
41 \mathrm{~N}, 178 \mathrm{~W}\end{array}$ & $\begin{array}{l}16 \\
15 \\
13\end{array}$ & $\begin{array}{l}8 \\
9 \\
8\end{array}$ & $\begin{array}{l}15 \\
15 \\
14\end{array}$ & $\begin{array}{l}8 \\
9 \\
8\end{array}$ \\
\hline $\begin{array}{l}43 \mathrm{~N}, 158 \mathrm{~W} \\
42 \mathrm{~N}, 158 \mathrm{~W} \\
41 \mathrm{~N}, 158 \mathrm{~W}\end{array}$ & $\begin{array}{l}14 \\
15 \\
15\end{array}$ & $\begin{array}{l}9 \\
9 \\
9\end{array}$ & $\begin{array}{l}14 \\
15 \\
16\end{array}$ & $\begin{array}{r}9 \\
10 \\
10\end{array}$ \\
\hline \multicolumn{5}{|c|}{ Southern region } \\
\hline $\begin{array}{l}31 \mathrm{~N}, 178 \mathrm{~W} \\
30 \mathrm{~N}, 178 \mathrm{~W} \\
29 \mathrm{~N}, 178 \mathrm{~W}\end{array}$ & $\begin{array}{l}13 \\
15 \\
13\end{array}$ & $\begin{array}{l}8 \\
8 \\
8\end{array}$ & $\begin{array}{l}15 \\
14 \\
14\end{array}$ & $\begin{array}{r}8 \\
9 \\
10\end{array}$ \\
\hline $\begin{array}{l}31 \mathrm{~N}, 158 \mathrm{~W} \\
30 \mathrm{~N}, 158 \mathrm{~W} \\
29 \mathrm{~N}, 158 \mathrm{~W}\end{array}$ & $\begin{array}{l}16 \\
16 \\
15\end{array}$ & $\begin{array}{r}10 \\
9 \\
9\end{array}$ & $\begin{array}{l}16 \\
17 \\
14\end{array}$ & $\begin{array}{r}10 \\
10 \\
9\end{array}$ \\
\hline
\end{tabular}

\section{The spectrum of turbulence in a stratified ocean}

A theoretical prediction of the turbulence spectrum in a stably stratified ocean was given by Phillips (1966). Energy is supposed to be received from larger scales of motion, modified by buoyancy, and passed on to smaller eddies until finally dissipated by viscosity. The range of the spectrum, where buoyancy forces are important, is called the "buoyancy subrange," and the range between the latter and the start of the viscous dissipation is called the "inertial subrange." Over the entire buoyancy-inertial range the spectrum is given by (Phillips, 1966)

$$
E(K)=A \epsilon_{0}^{\frac{2}{3}}\left(1+B N^{2} \epsilon_{0}^{-\frac{2}{3}} K^{-4 / 3}\right) K^{-5 / 3},
$$

where $\epsilon_{0}$ is the net rate of viscous dissipation, $N$ the Väisälä frequency, $A$ the universal Kolmogorov constant (1.44 \pm 0.07$)$, and $B$ a positive constant of order unity. The Väisälä frequency in the pycnocline has a

TABLE 2. Largest amplitudes of the perturbations.

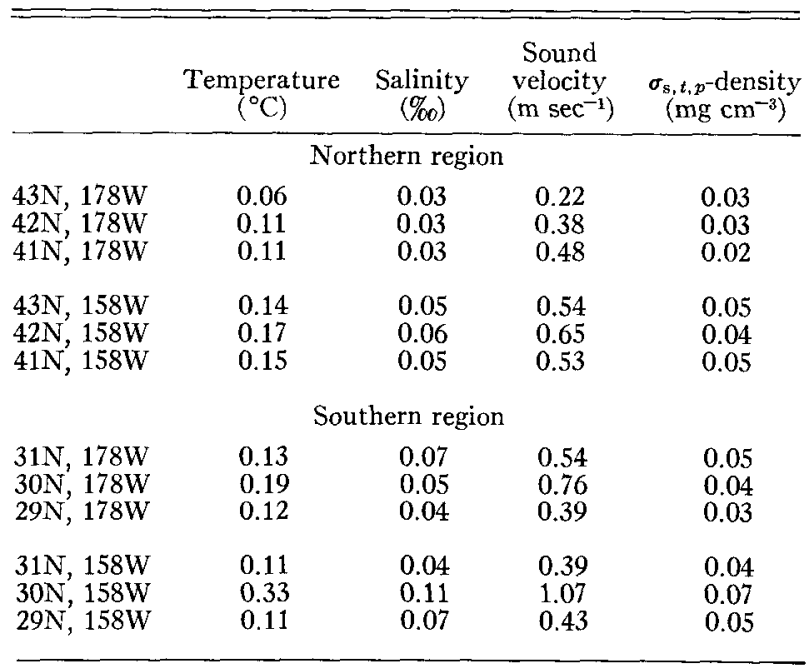


characteristic magnitude of $10^{-2} \mathrm{sec}^{-1}$, and in deep water it may be as low as $10^{-4} \mathrm{sec}^{-1}$. The numerical value for the net rate of viscous dissipation varies considerably; indeed, Grant et al. (1962) found it to lie between $1.5 \times 10^{-3}$ and $1 \mathrm{~cm}^{2} \mathrm{sec}^{-3}$, which covers a range of almost three orders of magnitude.

It is obvious from (5) that in the absence of stratification $(N \rightarrow 0)$ or at very high wavenumbers $(k \rightarrow \infty)$, the expression reduces to the classical $-5 / 3$ law. For very strong stratification $(N \rightarrow \infty)$ or at very low wavenumbers $(k \rightarrow 0), \mathrm{a}-3$ power law is obtained. Neither Väisälä frequency extreme is likely to be encountered over large areas of the ocean, and hence the spectral energy decay with increasing wavenumber should be following a power law with coefficients between -1.7 and -3 at low and intermediate wavenumbers.

The expression given in (5) refers to turbulent velocity fluctuations. A similar relationship can be derived for passive scalar variables transported by the velocity field (Lumley and Panofsky, 1964). Temperature and salinity in the subsurface layers of the ocean are examples of such variables. The constants in (5) will be different for each variable, however, because the rate of viscous dissipation differs, in general, from the rates of heat diffusion and salt diffusion (Phillips, 1966; Turner, 1965). Consequently, the bouyancy and inertial subranges of the velocity spectrum may differ from those of the temperature and salinity spectra. This is an important theoretical aspect; unfortunately, no comparative sets of oceanic measurements exist to investigate these differences quantitatively.

In the uppermost layers of the sea, energy exchange with the atmosphere is important. Here, temperature and salinity are not transported passively by the velocity field, but are modified by such processes as radiative heat transfer and the evaporation-precipitation regime. The details of these processes are very complicated and are not well understood at present. Their presence indicates, however, that the velocity, temperature and salinity spectra may differ from each other. Comparative sets of measurements in the ocean are urgently needed to make a quantitative assessment of the differences.

\section{Observed wavenumber spectra with respect to depth}

The following spectra are based on perturbation series, such as shown in Fig. 2, which are characterized by a zero mean value and a nonhomogeneous amplitude, depending upon depth. Because of the nonhomogeneous amplitude, ordinary spectrum analysis can yield only limited information on the statistical properties of the random process. Herein lies the difficulty of procedure. In principle, two approaches to the problem are available: 1) the nonhomogeneous record could be subdivided into homogeneous parts and the ordinary spectrum could be computed for each one, and 2) higher order spectra could be computed over the entire series. Either method requires about a hundred times as many data points for statistical reliability as are presently available. Nevertheless, it is of interest to take a more modest approach and to look briefly at the limited information that can be obtained from the ordinary spectrum determined over the 0-1500 $\mathrm{m}$ depth interval. The results, of course, describe the average properties of the random process over this particular interval, and should not be extrapolated to any other depth intervals. With this limitation, the pertinent questions to be asked are : 1) How does the spectral density decrease with increasing wavenumber? 2) Are there any preferred wavenumbers at which the spectral density is concentrated? 3) For the same variable, does the shape of the spectrum depend upon geographical location? 4) For different variables at the same station, are the 'spectral shapes the same?

The computations were carried out for the wavenumber range between 0 and 167 cycles per kilometer (c.p.km) with a resolution of 3.33 c.p.km, using the Tukey method (Blackman and Tukey, 1958). All spectra were corrected for binomial filtering and plotted on a $\log -\log$ scale. In each case, the number of data points was 501, and the degrees of freedom were 23.5.

The filtering scheme chosen prewhitens the spectrum in the low wavenumber region. By analyzing the prewhitened spectrum, and then correcting for the filter, the resulting spectral estimates are more reliable than those obtained by merely subtracting the overall mean.

Spectra were computed for temperature, salinity, sound velocity and density perturbations. Although the last two variables were not independently measured, but computed from the former, their inclusion here is of practical interest. Moreover, it is easier to interpret the sound velocity and density spectra directly than to deduce them from the spectra of temperature and salinity, since temperature and salinity perturbations are not coherent at all wavenumbers.

\section{a. Temperature perturbations}

The power density spectra for the vertical temperature perturbations are shown in Fig. 3, for different regions of the transition zone. The inset scales refer to the $95 \%$ confidence limits. The instrumental threshold accuracy for the spectra is $2 \times 10^{-8}\left({ }^{\circ} \mathrm{C}\right)^{2}(\text { c.p.km) })^{-1}$, which is about two orders of magnitude smaller than the measured power density levels. The power density of the temperature perturbations decreases with increasing wavenumber, rapidly at first and then more slowly. The decay coefficient is about $-8 / 3$ at low wavenumbers $(3-30$ c.p.km). Its value at high wavenumbers is less, but the magnitude is difficult to determine owing to the superimposed random fluctuations. There are several small peaks in the spectra, none of them significant at the $95 \%$ confidence level. For the range of scales investigated here (between 6 and 


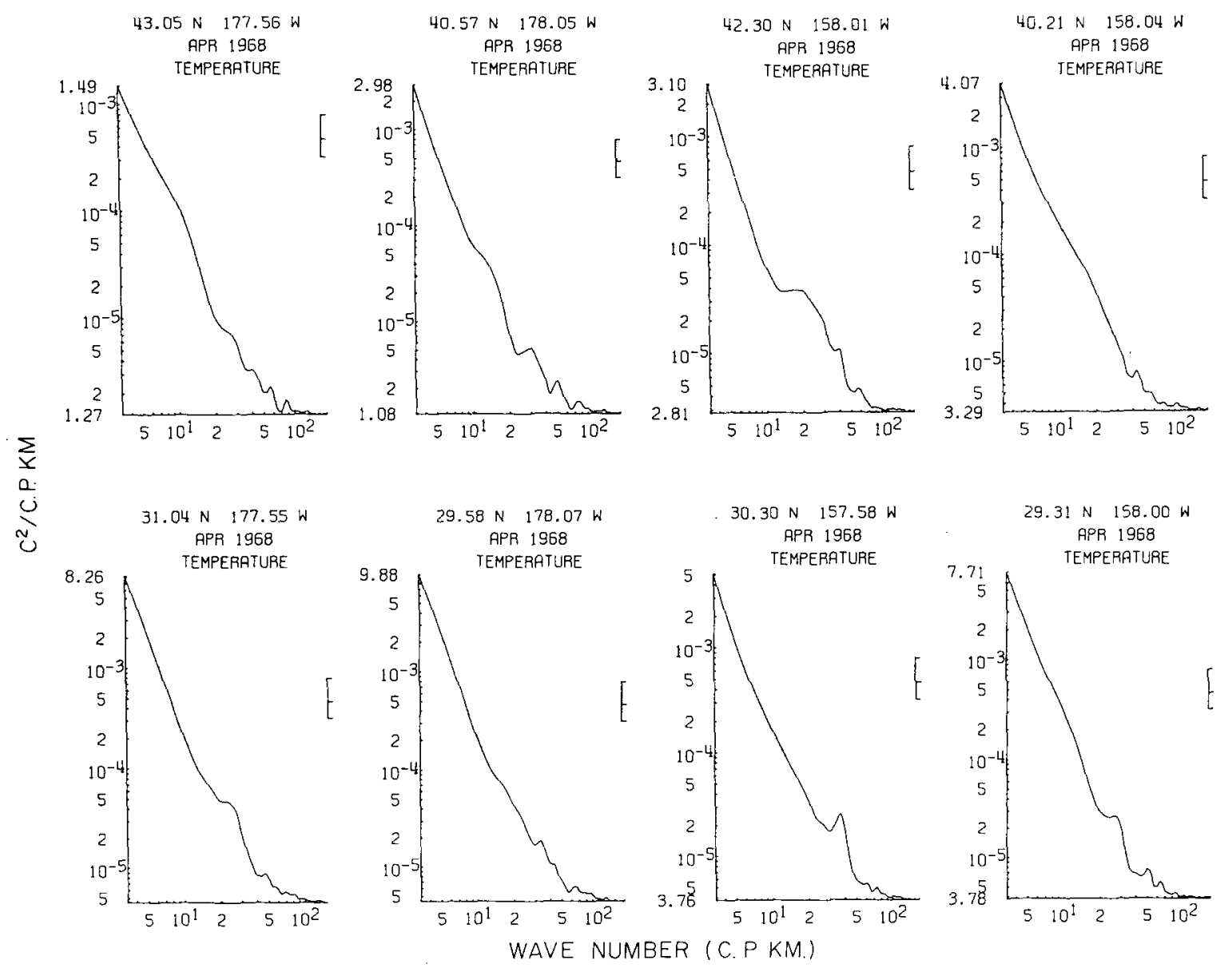

Fig. 3. Wavenumber spectra of temperature perturbations. Arrows indicate $95 \%$ confidence limits.

$30 \mathrm{~m}$ ), this indicates that there are no preferred vertical length scales at which the power of the temperature perturbations is concentrated. It is noteworthy that the shape of the spectrum is independent of geographical location, there being no significant differences between the northern, southern, eastern or western regions of the mid-Pacific transition zone. This suggests that the physical processes leading to random temperature perturbations must be similar over distances of the order of the width of the transition zone, which is $\sim 1000 \mathrm{~km}$.

\section{b. Salinity perturbations}

The power density spectra for the vertical salinity perturbations are shown in Fig. 4 for the same stations as in the case of temperature. The instrumental threshold accuracy for the spectra is here $3 \times 10^{-7}$ (per mille) ${ }^{2}$ (c.p.km) $)^{-1}$, which is encountered at the first two stations in the northern part of the transition zone near longitude $178 \mathrm{~W}$. At these two stations, the spectrum at wavenumbers $>40$ c.p.km is statistically unreliable. At all other stations, however, the spectrum is meaningful over the entire wavenumber range. The power density of the salinity perturbations decreases with increasing wavenumber in a way reminiscent of the temperature perturbations. At low wavenumbers (3-30 c.p.km), the decay coefficient varies between $-8 / 3$ and $-9 / 3$. As the wavenumber increases, the decay coefficient decreases, but its exact value at higher wavenumbers is difficult to obtain because of the random fluctuations superimposed upon the smooth shape of the spectrum (such as would be obtained by averaging over neighboring frequencies). The spectra of the salinity perturbations in the northern and southern regions of the mid-Pacific transition zone do not differ much from each other. Where differences occur, they are related to the position of the spectral peaks. Most of the peaks are statistically insignificant at the $95 \%$ confidence level. Significant peaks occur near wavenumbers of 60 and 80 c.p.km at some stations. This suggests that the power of the salinity perturbations is concentrated at preferred vertical length scales of 13 and $17 \mathrm{~m}$, in these cases. The reasons for the existence of such preferred scales and for their geographically intermittent character are not well understood at present. 


\section{c. Sound velocity perturbations}

Sound velocity was not measured directly by the STD-device, but was computed from the observed temperatures, salinities and pressures (depths) using Wilson's equation (Vigoureux and Hersey, 1962). In temperate and low latitudes, sound velocity perturbations are primarily a function of temperature. The power density spectrum of sound velocity perturbations can therefore be expected to resemble that of temperature perturbations. This agreement is fully borne out in Fig. 5, which, when compared to Fig. 3, shows hardly any differences in the shape of the spectrum or in the position of the peaks. The spectral shapes in different geographical regions of the mid-Pacific transition zone are essentially the same.

\section{d. Density perturbations}

Density was computed from the observed temperatures, salinities and pressures (depths) by conventional method and reduced to

$$
\sigma_{s, t, p}=\left(\rho_{s, t, p}-1\right) 10^{3},
$$

where $\sigma_{s, t, p}\left(\mathrm{mg} \mathrm{cm}^{-3}\right)$ is the reduced density in situ
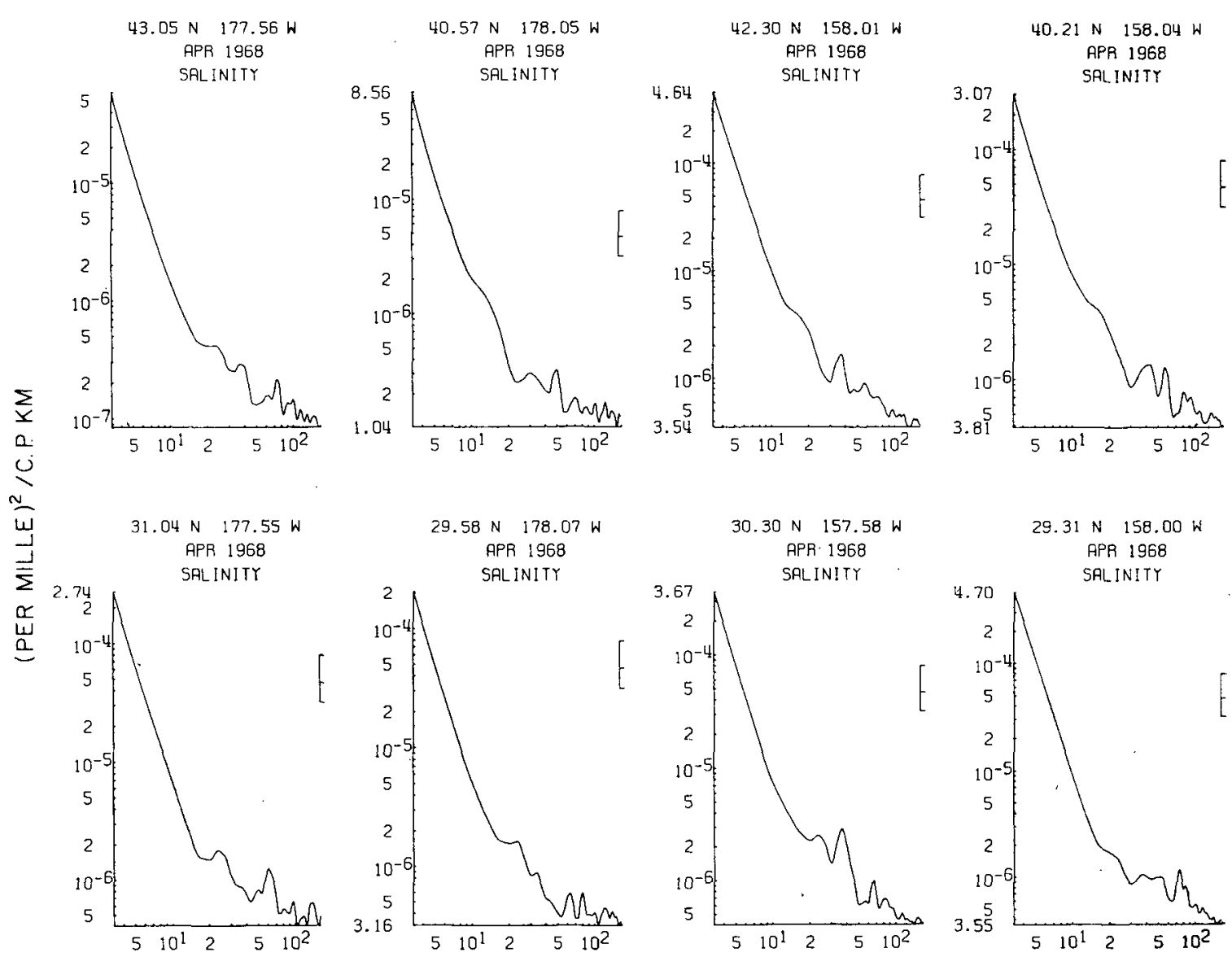

\section{The station-to-station coherence of temperature and} of salinity perturbations was computed for three mepower spectra for the perturbations of the reduced density in situ are shown in Fig. 6. The threshold accuracy for these spectra is $\left.3 \times 10^{-7}(\mathrm{mg} \mathrm{cm})^{-3}\right)^{2}$ (c.p.km) ${ }^{-1}$. To some extent, the shape of the density in situ perturbation spectrum resembles that of salinity. There is a decrease of power with increasing wavenumber, the decrease being stronger at low than at high wavenumbers. Superimposed upon the smooth curve (such as would be obtained by averaging over neighboring frequencies) are random fluctuations, notably at wavenumbers $>40$ c.p.km. In most cases, the added peaks and troughs are statistically insignificant at the $95 \%$ confidence level.

Perturbations of the vertical density gradient are important in the dynamics of ocean currents (Eckart, 1960). The present accuracy of the salinity sensor of the STD-device is such, however, that these perturbations cannot be determined in a reliable manner.

\section{Meridional coherence of the perturbations}
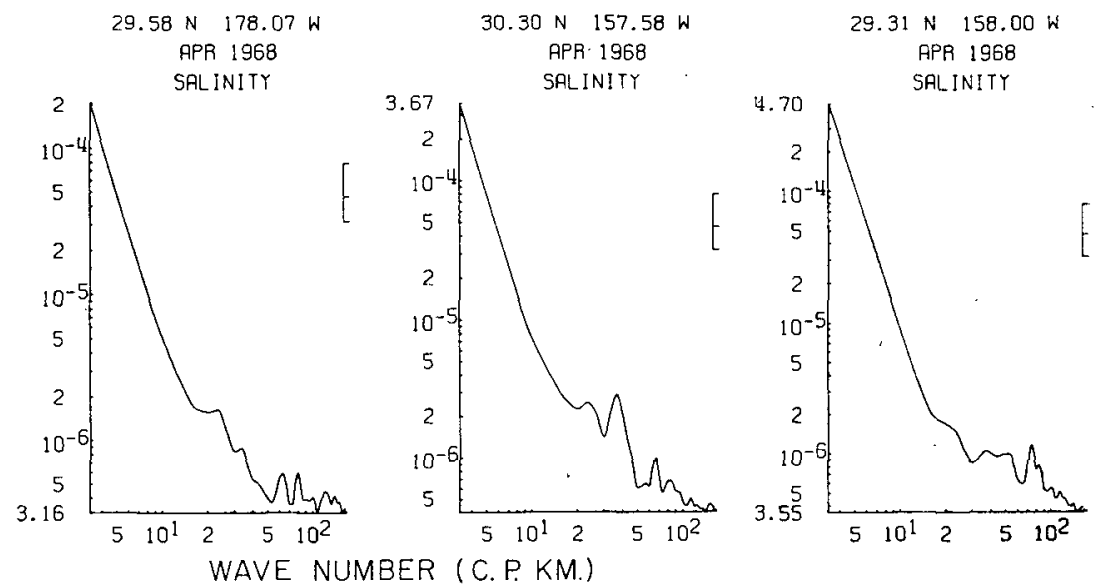

FIG. 4. Wavenumber spectra of salinity perturbations. Arrows indicate $95 \%$ confidence limits. 


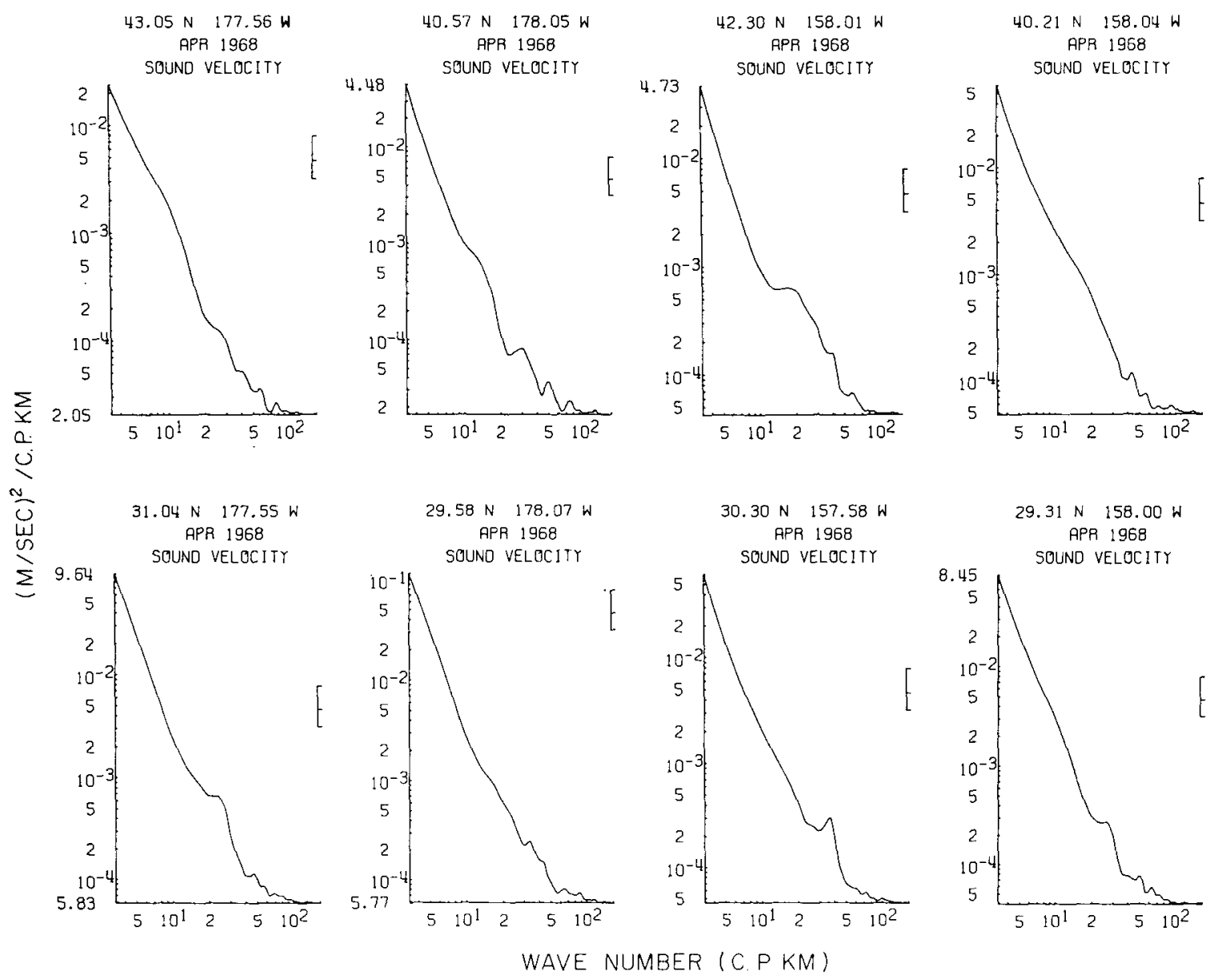

FIG. 5. Wavenumber spectra of sound velocity perturbations. Arrows indicate $95 \%$ confidence limits.

ridional sections. At longitude $178 \mathrm{~W}$, the stations were $37 \mathrm{~km}$ apart, while at longitudes 158 and $168 \mathrm{~W}$, they were $55 \mathrm{~km}$ apart. Marginal statistically significant coherence (between 0.5 and 0.6 ) at low wavenumbers (from 20-40 c.p.km) was found near the northern boundary of the transition zone; elsewhere, there was no such coherence. This seems to indicate that perturbations with vertical length scales $<25 \mathrm{~m}$ are rather local. These findings are in agreement with LaFond's (1963) studies of the horizontal dimensions of the temperature structure.

\section{Coherence between perturbations of temperature and salinity}

The coherence between temperature and salinity perturbations at given stations is shown in Fig. 7. The coherence spectra are similar in the different regions of the mid-Pacific transition zone. In all cases, there is statistically significant coherence at wavenumbers between 20 and 50 c.p.km. The exact reason for this consistent feature is not known. A possible explanation is that the mechanisms leading to the formation of tem- perature and salinity perturbations depend upon wavenumber. At low wavenumbers, buoyancy forces dominate; the vertical density stratification is normally stable and tends to remain stable after perturbations by internal waves and other types of motion. Because of the vertical stability constraint (which is an observational fact), temperature perturbations of one sign will be associated with salinity perturbations of the opposite sign. If this were not so, vertical instabilities at low wavenumbers would be common, which is contrary to observations. At high wavenumbers, on the other hand, buoyancy forces become subordinate to heat and salt diffusion effects, and the temperature and salinity perturbations may be caused by diffusion. Since the rates of heat and salt diffusion are not necessarily the same (Turner, 1965), the formation of "salt fingers" is not always accompanied by the generation of "heat fingers;" hence, there need not be a definite relationship between temperature and salinity perturbations. At this stage, the above discussion is only a conjecture, of course, that is in need of experimental verification. 


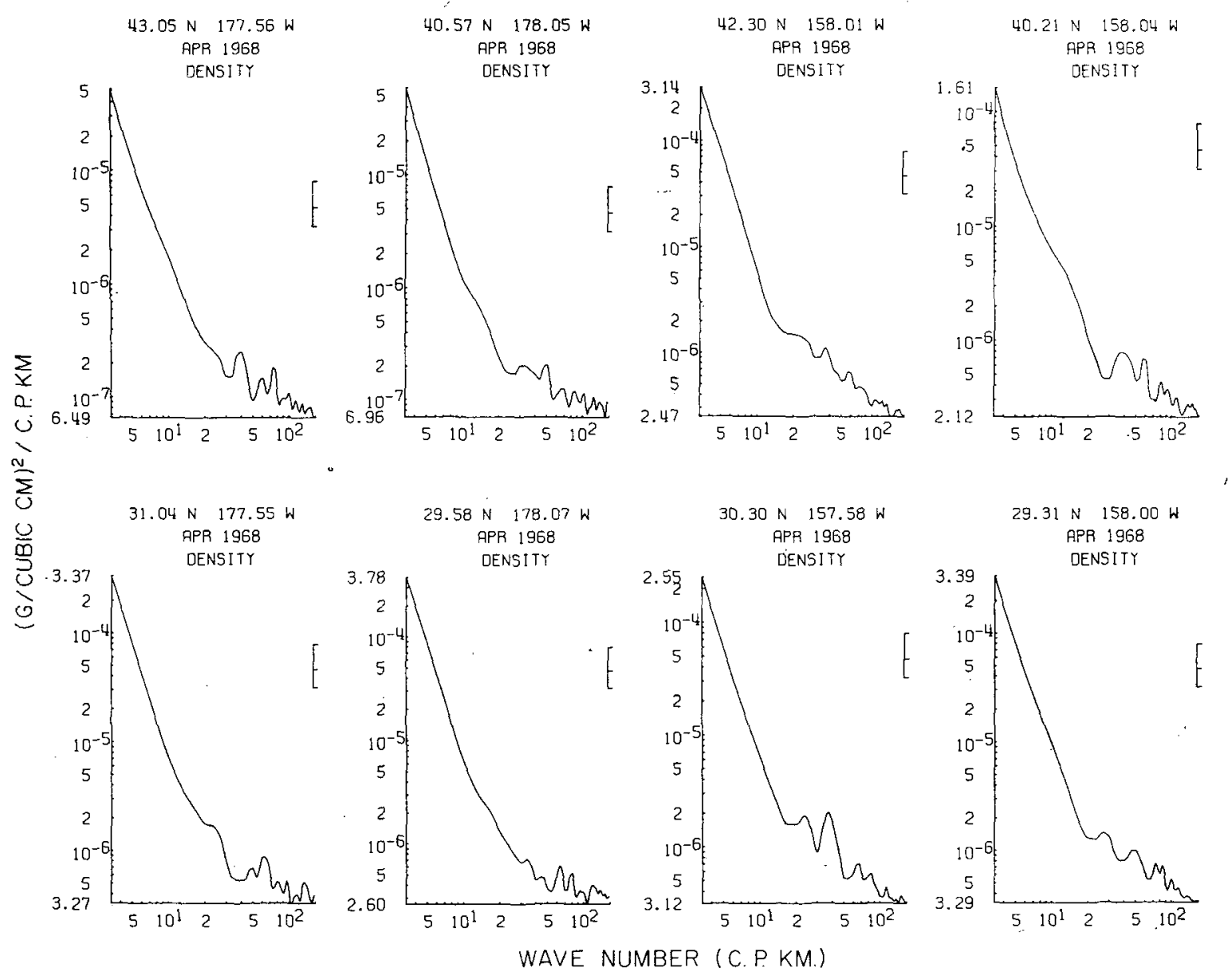

FIG. 6. Wavenumber spectra of $\sigma_{s, t, p}$-density perturbations. Arrows indicate $95 \%$ confidence limits.

\section{Conclusions}

The following conclusions can be drawn from an analysis of temperature and salinity measurements against depth, as obtained by the STD-device in the
mid-Pacific transition zone between the subarctic and subtropical water masses:

1) The highly complicated observed vertical temperature and salinity profiles can be partitioned into
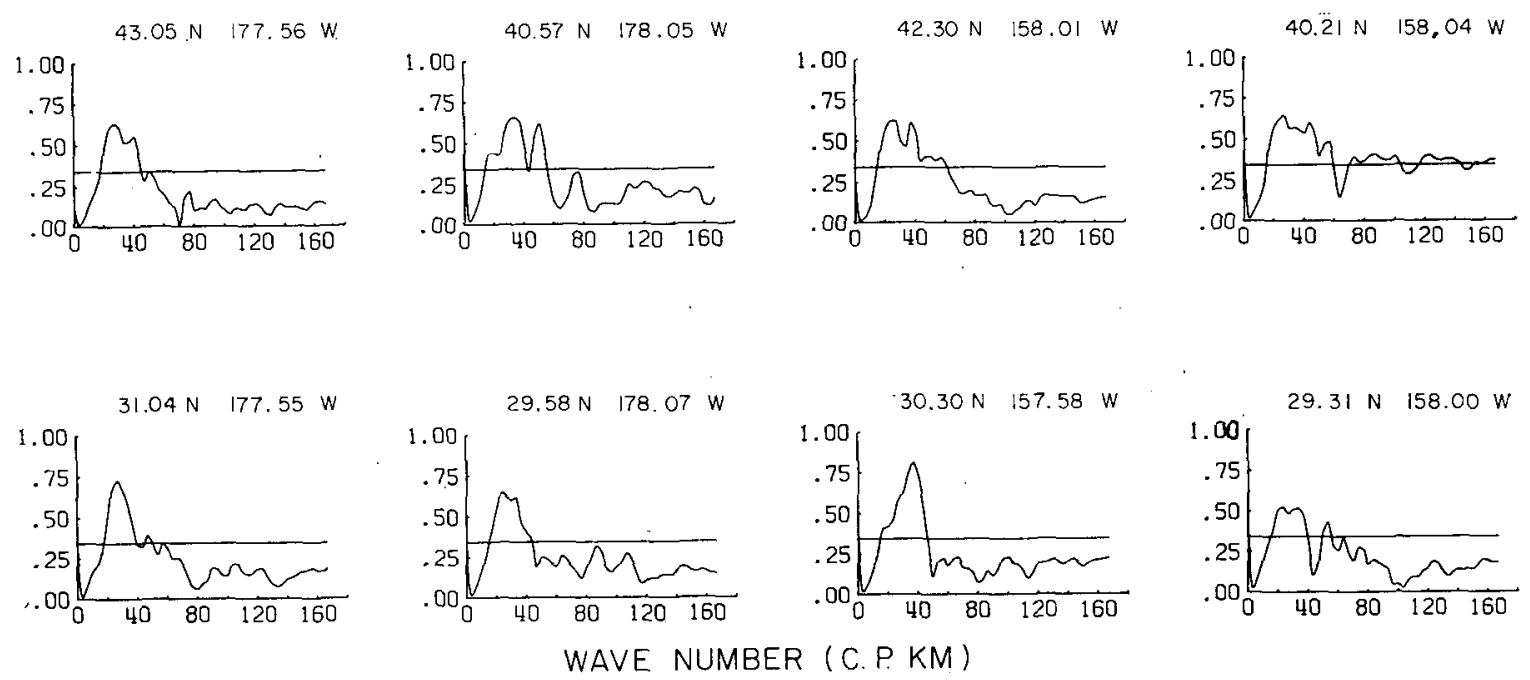

FIG. 7. Wavenumber spectra of the coherence between temperature and salinity perturbations at the same station. The dashed line indicates the $95 \%$ confidence limit. 
simple mean and perturbation profilès by suitable filtering techniques. The choice of the particular filter involves a compromise between the degree of filtering and the loss of original information that can be tolerated.

2) The amplitude of the temperature and salinity perturbations depends upon depth and the proximity of water mass boundaries. The largest amplitudes are found in the thermocline and halocline and near the northern and southern limits of the transition zone. Typical maximum amplitudes of the temperature perturbations are $0.1-0.2 \mathrm{C}$. Typical maximum amplitudes of the salinity perturbations are $0.05-0.10 \%$.

3) The power density spectra over the wavenumber range between 0 and 167 c.p.km show a characteristic decrease of the power density with increasing wavenumber. The decay coefficient at low wavenumbers varies typically between $-8 / 3$ and $-9 / 3$ and is in agreement with the theory of turbulence in a stably stratified medium (Phillips, 1966).

4) For a given variable, the power density spectra in different geographical regions of the transition zone have a similar shape.

5) The perturbations are generally incoherent over distances of the order of $50 \mathrm{~km}$.

6) For a given station, temperature and salinity perturbations are coherent at low wavenumbers and incoherent at high wavenumbers. This may indicate that processes leading to large-scale vertical perturbations differ from those leading to small-scale perturbations.

Acknowledgments. I am indebted to M. Rattray and S. R. Murphy for fruitful discussions. D. Bendiner carried out the calculations and programming operations. The scientific and operational crew of the Thomas G. Thompson, Capt. Vern Wyatt commanding, are to be commended for outstanding performance.

This is contribution No. 0542 from the Department of Oceanography, University of Washington, Seattle, Washington. The research reported herein was supported by the Office of Naval Research under Contract N 00014-67-A0103-0014 Project 083012.

\section{REFERENCES}

Blackman, R. B., and J. W. Tukey, 1958: The Measurement of Power Spectra. New York, Dover, 190 pp.

Bolgiano, R., 1962: Structure of turbulence in stratified media. J. Geophys. Res., 67, 3015-3023.

Cairns, J. L., and E. C. LaFond, 1966: Periodic motions of the seasonal thermocline along the southern California coast. J. Geophys. Res., 71, 3903-3915.
Eckart, C., 1960: Hydrodynamics of Oceans and Atmospheres. New York, Pergamon, $290 \mathrm{pp}$.

F jeldstad, J. E., 1964: Internal waves of tidal origin. Geofys. Publikasjoner, 25, 2-73.

Grant, H. L., R. W. Stewart and A. Moilliet, 1962: Turbulence spectra from a tidal channel. $J$. Fluid Mech., 12, 241-263.

Koizumi, M,, 1955 : Researches on the variations of oceanographic conditions in the region of the ocean weather station "Extra" in the North Pacific (I). Papers Meteor. Geophys. Tokyo, 6, 185-201.

LaFond, E. C., 1962: Temperature structure of the upper layer of the sea and its variation in time. Temperalure-Its Measurement in Science and Industry, Vol. 3, New York, Reinhold, 751-677.

__ 1963: Detailed temperature structures of the sea off Baja California. Limnology Oceanogr., 8, 417-425.

Lee, W. H., and C. S. Cox, 1966: Time variation of ocean temperaature and its relation to internal waves and oceanic heat flow measurements. J. Geophys. Res., 71, 2101-2111.

Lovett, J. R., 1968: Vertical temperature gradient variations related to current shear and turbulence. Limnology Oceanogr., $13,127-142$.

Lumley, J. L., 1964 : The spectrum of nearly inertial turbulence in a stably stratified fluid. $J$. Almos. Sci., 21, 99-102.

- - and H. A. Panofsky, 1964: The Structure of Atmospheric Turbulence. New York, Interscience, $239 \mathrm{pp}$.

MacCready, P. B., 1962: The inertial subrange of atmospheric turbulence. J. Geophys. Res., 67, 1051-1059.

Munk, W. H., and N. Phillips, 1968: Coherence and band structure of inertial motion in the sea. Rev. Geophys., 6, 447-472.

Myrup, L. O., 1968: Atmospheric measurements of the buoyant subrange of turbulence. I. Amos. Sci., 25, 1160-1164.

Phillips, O. M., 1966: The Dynamics of the Upper Ocean. Cambridge University Press, $261 \mathrm{pp}$.

Rattray, M., 1964: Time-dependent motion in an ocean : A unified two-layer, beta plane approximation. Studies in Oceanography, Dedicated to Professor Hidaka in Commemoralion of His Sixieth Birthday, University of Tokyo Press, $560 \mathrm{pp}$.

- , and R. L. Charnell, 1966: Quasi-geostrophic free oscillations in enclosed basins. J. Marine Res., 24, 82-102.

Reid, J. L., 1956: Observations of internal tides in October 1950. Trans. Amer. Geophys. Union, 37, 278-286.

Roden, G. I., 1963: Shallow temperature inversions in the Pacific Ocean. J. Geophys. Res., 69, 2899-2914.

- - 1970: Aspects of the mid-Pacific transition zone. J. Geophys. Res., 75, 1097-1109.

Saffman, P. G., 1968: Lectures on homogeneous turbulence. Topics in Nonlinear Physics, New York, Springer Verlag, $724 \mathrm{pp}$.

Stewart, R. W., and H. L. Grant, 1962: Determination of the rate of dissipation of turbulent energy near the sea surface in the presence of waves. J. Geophys. Res., 67, 3177-3180.

Tabata, S., 1965 : Variability of oceanographic conditions at ocean station "P" in the Northeast Pacific Ocean. Trans. Roy. Soc. Canada, 3, 367-418.

Turner, J. S., 1965: The coupled turbulent transports of salt and heat across a sharp density interface. Intern. J. Heat Mass Transfer, 8, 759-767.

Vigoureux, P., and J. B. Hersey, 1962 : Sound in the sea. The Sea, Vol. 1, New York, Interscience, $864 \mathrm{pp}$.

Webster, F., 1968: Observations in inertial period motions in the deep sea. Rev. Geophys., 6, 473-490. 